\title{
BMJ Open Study protocol of the internet user Cohort for Unbiased Recognition of gaming disorder in Early adolescence (iCURE), Korea, 2015-2019
}

Hyunsuk Jeong, ${ }^{1}$ Hyeon Woo Yim, ${ }^{1}$ Sun-Jin Jo, ${ }^{1}$ Seung-Yup Lee, ${ }^{2}$ Eunjin Kim, ${ }^{1}$ Hye Jung Son, ${ }^{1}$ Hyun-ho Han, ${ }^{1}$ Hae Kook Lee, ${ }^{2}$ Yong-Sil Kweon, ${ }^{2}$ Soo-young Bhang, ${ }^{3}$ Jung-Seok Choi, ${ }^{4}$ Bung-Nyun Kim, ${ }^{5}$ Douglas A Gentile, ${ }^{6}$ Marc N Potenza ${ }^{7}$

To cite: Jeong H, Yim HW, Jo S-J, et al. Study protocol of the internet user Cohort for Unbiased Recognition of gaming disorder in Early adolescence (iCURE), Korea, 2015-2019. BMJ Open 2017;7:e018350. doi:10.1136/ bmjopen-2017-018350

- Prepublication history for this paper is available online. To view these files please visit the journal online (http://dx.doi. org/10.1136/bmjopen-2017018350).

Received 23 June 2017 Revised 27 July 2017 Accepted 15 August 2017

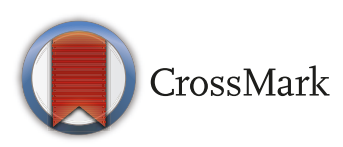

For numbered affiliations see end of article.

Correspondence to Dr Hyeon Woo Yim; y1693@catholic.ac.kr

\section{ABSTRACT}

Introduction In 2013, the Diagnostic and Statistical Manual of Mental Disorders, Fifth Edition (DSM-5) proposed nine internet gaming disorder (IGD) diagnostic criteria as a condition warranting further empirical and clinical research. The aim of this study is to clarify the natural and clinical courses of IGD proposed DSM-5 in adolescents and to evaluate its risk and protective factors.

Methods and analysis The Internet user Cohort for Unbiased Recognition of gaming disorder in Early Adolescence (iCURE) study is an ongoing multidisciplinary, prospective, longitudinal cohort study conducted in 21 schools in Korea. Participant recruitment commenced in March 2015 with the goal of registering 3000 adolescents. The baseline assessment included surveys on emotional, social and environmental characteristics. A parent or guardian completed questionnaires and a structured psychiatric comorbidity diagnostic interview regarding their children. Adolescents with the Internet Game UseElicited Symptom Screen total scores of 6 or higher were asked to participate in the clinical diagnostic interview. Two subcohorts of adolescents were constructed: a representative subcohort and a clinical evaluation subcohort. The representative subcohort comprises a randomly selected $10 \%$ of the iCURE to investigate the clinical course of IGD based on clinical diagnosis and to estimate the false negative rate. The clinical evaluation subcohort comprised participants meeting three or more of the nine IGD criteria, determined by clinical diagnostic interview, to show the clinical course of IGD. Follow-up data will be collected annually for the 3 years following the baseline assessments. The primary endpoint is 2-year incidence, remission and recurrence rates of IGD. Cross-sectional and longitudinal associations between exposures and outcomes as well as mediation factors will be evaluated.

Ethics and dissemination This study is approved by the Institutional Review Board of the Catholic University of Korea. Results will be published in peer-reviewed journals.

Trial registration number ClinicalTrials.gov (identifier: NCT02415322).

\section{Strengths and limitations of this study}

- Internet gaming disorder (IGD) was assessed by using both polychotomous and dichotomous tools based on the nine Diagnostic and Statistical Manual of Mental Disorders, Fifth Edition (DSM-5) IGD criteria. In addition, we are conducting structured interviews using Diagnostic Interview Schedule for Children, Version 4 of the parents or guardians to assess participant psychiatric comorbidities.

- This study is that the clinical diagnosis of IGD based on the DSM- 5 is performed by qualified mental health specialists.

- It is not a representative sample because the registration was conducted among the schools in the specific area.

- The participants were included only third-grade, fourth-grade and seventh-grade students.

\section{INTRODUCTION}

Internet games are popular entertainment in modern society, with a variety of people of different ages participating in gaming. Internet game play rates and sales are increasing. ${ }^{1}$ Players choose to play games for many reasons, including fun, recreation, coping with stress, sociability and escaping real life. ${ }^{2-4}$ The gaming research literature has reported both positive and negative effects on players. Playing games promotes problem solving and visual, motor and spatial skills, and it fosters interaction with friends. ${ }^{5}$ Although there are several positive effects of playing games for adolescents, there can also be negative effects, such as the possibility of problematic or addictive gaming. ${ }^{6}$ Gaming has been associated with inattention and hyperactivity, aggressive behaviours, negative emotions including depression, ${ }^{7} 8$ low self-esteem, social anxiety, loneliness ${ }^{9}$ and low psychological well-being. ${ }^{10}$ 
South Korea is one of the most highly digitalised societies in the world. The internet penetration rate in South Korea exceeded $75 \%$ in $2011 .{ }^{11}$ More than half of people in their $50 \mathrm{~s}$ and almost $100 \%$ of teenagers use the internet in their daily lives. ${ }^{12}$ Korea has developed a computer industry with internet use reaching over $50 \%$ of the population. Currently, in Korea, 25 million among 45 million citizens use the internet, and 14.4 million homes are equipped with internet access. In addition to home assess, many people visit 'PC rooms', a type of gaming centre with a broadband internet network that is equipped with the best performance computers in Korea. $\mathrm{PC}$ rooms became popular following the release of the PC game StarCraft in 1998, with an estimated 25000 PC rooms nationwide in 2014. 'PC rooms' have no any age restrictions on accessibility, and many adolescents often play online games. ${ }^{13}$ A 2010 nationwide survey regarding internet addiction showed that $8.0 \%$ of the Korean population and $12.4 \%$ of adolescents were addicted to internet use, as assessed via the self-administered Korean Scale for Internet Addiction(K-scale). ${ }^{12}$ This prevalence is a little higher than has been found in other countries, such as Singapore $(8.7 \%),{ }^{14}$ the Netherlands $(6.8 \%),{ }^{15}$ the USA $(8.5 \%)^{16}$ and China $(10.8 \%) .{ }^{17}$ The higher prevalence in Korea could be expected, given that it is a country with such broad and easy access. Although the number of internet game users has increased dramatically over the last decade, the phenomenon of internet gaming addiction is not yet well understood, and research on its aetiology and natural history is still in its infancy. Studies have used various tools to estimate the prevalence of internet gaming disorder (IGD), but the lack of common diagnostic criteria for IGD has created difficulties in comparing studies.

In May 2013, IGD was included in Section III of the Diagnostic and Statistical Manual of Mental Disorders, Fifth Edition (DSM-5) as a condition warranting further study. ${ }^{18}$ This marked the first occasion of IGD being formally recognised as a mental health disorder and suggested IGD classification criteria have paved the way for measuring IGD in a consistent manner. These suggestions enable more direct comparisons of findings and may be used to eventually reach a consensus on the status of the disorder. ${ }^{19}$ The DSM-5 proposes nine criteria for IGD, and it states explicitly that the specific criteria and the threshold for diagnosis require systematic investigation and empirical validation before their wide-scale adoption. The proposed criteria apply only to internet gaming and not general internet use, which can differ in terms of presenting symptoms, aetiology, comorbidities, course and treatment. Empirical data are required to ascertain the extent to which these criteria apply to various populations and to aid diagnosis.

However, the classification and rationale for DSM-5 IGD as a mental health disorder have been controversial. ${ }^{20} \mathrm{~A}$ previous study was proposed the possibilities that gaming behaviour can be common behaviour or leisure activity. Excessive involvement on gaming per se might reflect engagement, passion or coping. ${ }^{21}$ Excessive behaviours on gaming can be transient for many adolescents and we will look into whether excessive behaviour on gaming is episodic or steady through the longitudinal observation. It is also argued that several IGD criteria such as withdrawal and tolerance are often inappropriate to be put into the conceptualisation of behavioural addiction and difficult to apply convincingly and measure in relation to behaviours. ${ }^{22}$ In addition, a previous study did not support the hypothesis that the nine DSM-5 IGD criteria had the same validity for the diagnosis. ${ }^{23}$ To this end, we registered and began a prospective school-based cohort study of adolescents to provide evidence regarding the IGD diagnostic criteria by clarifying the characteristics of IGD's natural history. Given the broad discussion of a replicability 'crisis' in many sciences, it is valuable to document a priori our research methods, goals and planned approach.

\section{Aims}

The aim of the study is to clarify the natural and clinical courses of IGD based on the DSM- 5 diagnostic criteria, to provide evidence for empirical validation of the suggested IGD diagnostic criteria and to further evaluate the aetiological risk and predictive factors in adolescents.

\section{METHODS \\ Study design and setting}

The Internet user Cohort for Unbiased Recognition of gaming disorder in Early Adolescence (iCURE) study is a multidisciplinary, prospective, longitudinal cohort study of 3000 adolescents, conducted in Korea, with four waves of annual data collection currently planned. The study is being conducted in Seoul and Uijeongbu, Gyeonggi Province, with third-grade, fourth-grade and seventh-grade students in 6 primary schools and 19 secondary schools participating. Participation requires consent from the participants as well as written parental consent following explanation of the nature of the principles of research, including confidentiality and the freedom of choice to participate. The study was fully reviewed and approved by the Institutional Review Board of the Catholic University of Korea (MC140NM10085) and was conducted in accordance with the Declaration of Helsinki.

\section{Pretest and pilot test of methods}

We validated the feasibility of the study before beginning data collection by performing a pretest and a pilot test. For the pretest, we recruited a convenience sample of 25 third-grade and fourth-grade students to test the understandability of the questionnaires for this age group of students. We performed a pilot test to verify that the overall survey system worked well, with participants composed of one school class of 35 students. They completed all baseline measures which were recruited outside of the sampling frame. 


\section{Recruitment}

Participant recruitment commenced in March 2015. Recruitment took place at the school level. We sent official letters to all primary and secondary schools in three educational districts to invite their participation. After school principles provided consent for their schools to participate, the parents or guardians were contacted to obtain consent for their children to participate in the study. A total of 21 schools (6 elementary and 19 secondary) participated in the study, out of 258 schools (163 elementary and 95 secondary) in the three districts. In order to encourage participation in this research, the research team members visited the 21 participating schools to provide a seminar that presented the aims of the study and provided additional information about the research project to teachers and parents. The finial enrolment is expected to be 3000 adolescents. Three regional hospitals were designated in the three areas where the participating schools are located to provide treatment options for adolescents experiencing IGD.

\section{Inclusion and exclusion criteria}

The inclusion criteria were that the participants were third-grade, fourth-grade or seventh-grade students at baseline. We required that each student participant had a parent or guardian who also participated in the study. The exclusion criteria included lack of competence in the Korean language by the parent or guardian and intellectual disability of the adolescent.

\section{Data collection}

The participants completed questionnaires at baseline and will again annually for the following 3years. The baseline assessments started in May 2015. We are collecting data from multiple informants, including students' self-report questionnaires, clinical diagnostic interviews of the students, parents' or guardians' self-report questionnaires and structured clinical interviews of the parents or guardians regarding any psychiatric comorbidity in their children.

\section{Follow-up and retention strategy}

In an effort to maintain contact with iCURE study participants, we will send individual reports annually via registered mail, and we will periodically send text messages about general contents via cell phone to the participants. Once a year, we will send each participant's survey results to their parents via registered mail, which will be called a 'mental health signal'. This will include the mental health status regarding symptoms of internet game addiction, depression, anxiety and attention-deficit hyperactive disorder (ADHD), marked as red, yellow and green traffic signals, indicating symptom severity as assessed by both the self-report survey and the clinical diagnosis. We will also provide referrals for treatment availability.

During this study, we are operating a 24 hours counselling helpline for the parents and guardians. A clinical psychologist with more than 10 years of clinical experience specialising in children and adolescents is conducting telephone consultations. This telephone counselling is free and anonymous, providing parents with general counselling about their children, as well as specific counselling regarding gaming addiction.

\section{Students' self-report questionnaires}

Students' data collection is conducted at the schools during school hours. The seventh-grade students complete questionnaires using a web-based self-administration method. The third-grade and fourth-grade students complete the questionnaires in a class setting; a research assistant reads the questions aloud, following a standard script, to aid comprehension and diminish time demands. In later waves, all students will complete the questionnaires on their own, with a supervising research assistant available to answer questions. During the baseline assessment, the questionnaires took approximately 90-120 min to complete; later waves will take approximately $45-80 \mathrm{~min}$. The student data collection at later waves will also be conducted at the schools during school hours. For students who transfer to other schools, arrangements will be made to complete the follow-up questionnaires, either by visiting their homes or via website access.

\section{Interviewing parents}

At baseline, each parent or guardian completed a questionnaire and the Diagnostic Interview Schedule for Children, Version 4 (DISC-IV). The DISC-IV is a highly structured diagnostic interview used to assess psychiatric disorders in children and adolescents. We are including 7 of the 34 diagnostic assessments: generalised anxiety disorder, separation anxiety, obsessive-compulsive disorder, major depression disorder, attention deficit disorder and ADHD, oppositional defiant disorder and conduct disorder.

The DISC-IV was administered by trained interviewers at the participants' homes or at a private space at the school, based on the participant's preference. The parent questionnaire contained demographic questions and took 20-30 min to complete, and the DISC-IV took 40-90 min. Parent or guardian survey is conducting only at baseline.

\section{Second-stage clinical diagnosis by mental health professionals}

The clinical diagnostic interviews for IGD were conducted face-to-face by five psychiatrists and four master's degreelevel clinical psychologists with at least 5years of training and clinical experience. The interviews assessed participants based on the nine DSM-5 IGD criteria, in accordance with the international consensus for IGD represented in the DSM-5. ${ }^{19}$ Diagnoses were based on clinically significant functional impairments that substantially derived from gaming and not from any other psychiatric illnesses. Any psychiatric comorbidities were also thoroughly evaluated by the psychiatrists using the semistructured tool of the Kiddie-Schedule for Affective Disorders and Schizophrenia-Present and Lifetime Version-Korean version, which has been previously validated in Korea. ${ }^{24}$ Global functioning was assessed using the Children's Global Assessment Scale, based on the child's 
worst level of emotional and behavioural functioning in the past 3 months.

The criterion for a second-stage clinical diagnosis is an Internet Game Use-Elicited Symptom Screen (IGUESS) total score of 6 or higher, either at baseline or at the follow-up waves. Additionally, random selection of $10 \%$ of the full cohort, stratified by the school, completed the clinical diagnostic interviews at baseline and the further follow-up waves. Anyone who responds insincerely with marking all of the same numbers to the IGUESS at every wave will be excluded to rule out false negatives. Anyone who reports a suicidal experience during the past year at any wave will be given diagnostic interviews. The clinical diagnostic interviews are being conducted 1 week after completion of the baseline study. The interviews are being conducted confidentially in private spaces at the participants' schools.

\section{Subcohorts}

Two subcohorts have been constructed. A representative subcohort consists of a randomly selected $10 \%$ of adolescents in the cohort. They will participate in the clinical diagnostic interview every year to estimate the false negative rate and to evaluate the clinical course of DSM-5-defined IGD based on clinical diagnosis. Additionally, a clinical evaluation subcohort comprised those meeting three or more of the nine IGD criteria, as determined by the clinical diagnostic interview at baseline and at each follow-up wave. The reason why they were selected as a clinical evaluation subcohort was to observe the natural history or clinical course of them even though they did not reach the DSM-5 IGD diagnostic criteria. Following up this subcohort may reveal whether they actually turned out to be IGD or normal behaviour. The interviews will be conducted annually for 3 years to observe the clinical history, remission and recurrence rates.

\section{Measurement}

The measurements using iCURE are presented in table 1 .

\section{Demographic information}

Demographic characteristics were assessed at baseline. Sex, date of birth, family composition and number of siblings were obtained from the student assessment, and parental education level, socioeconomic status, parental marital status, parental education level and parental job were obtained from the parent or guardian assessments.

\section{Internet, gaming and smartphone use}

Comprehensive assessments of participants' internet, gaming and smartphone usage were performed, including questions regarding the first time of exposure, the average daily time spent online and gaming-related questions, such as online gaming type preference and weekly playing time. We classified online games as roleplaying games, shooter games, simulation games, arcade games and unknown.
Regulated usage of smartphones or games

We assess school policies on possession and usage of smartphones and domestic regulations of smartphone or game time.

\section{Internet Addiction Test (IAT)}

Young's IAT consists of 20 items rated on a 5-point Likert scale from rarely (1) to always (5), with higher scores indicating more severe internet addiction. ${ }^{25}$

\section{Korean Scale for Internet Addiction}

To assess internet addiction, we use the K-scale short form for adolescents, which were developed in Korea. It comprised 20 items rated on a 4-point Likert scale, from 1 (not at all) to 4 (always). ${ }^{26}$

\section{Internet Game Use-Elicited Symptom Screen}

This instrument was created based on the nine DSM-5 IGD criteria. Students are instructed to respond based on their gaming behaviour within the last 12 months, with each item rated on a 4-point scale: $1=$ strongly disagree, $2=$ somewhat disagree, $3=$ somewhat agree, $4=$ strongly agree.

Self-reported version of structured clinical interview for Internet Gaming Disorder (SR-IGD)

We use the SR-IGD to assess IGD with respect to occurrences over the past 6 months. The assessment uses yes-or-no questions. ${ }^{27}$

\section{Short version of the Smartphone Addiction Scale (SAS-SV)}

We assessed smartphone addiction using the SAS-SV. The SAS-SV addresses the following five content areas with respect to the previous year: (1) daily-life disturbance, (2) withdrawal, (3) cyberspace-oriented relationship, (4) overuse and (5) tolerance. It contains 10 items rated on a dimensional scale ( $1=$ strongly disagree to $6=$ strongly agree). The total scores range from 10 to 60 , with the higher scores indicating greater degrees of smartphone addiction. ${ }^{28}$

\section{Children's Depression Inventory (CDI)}

The CDI is a 27-item, self-rated, symptom-oriented scale suitable for youths aged 7-17 years. It assesses cognitive, affective, somatic and behavioural symptoms, with items scored from 0 to 2 , where 0 means the symptom is not present, 1 means the symptom is present and mild and 2 means the symptom is present and marked. ${ }^{29}$

\section{Trait Anxiety Inventory for Children (TAIC)}

To examine trait anxiety, we used the Korean translation $^{30}$ of the TAIC, which is part of the State-Trait Anxiety Inventory for Children developed by Spielberger and colleagues. ${ }^{31}$ The Korean TAIC is a 20 -item inventory that asks respondents to indicate how frequently, on a 3 -point scale ( $1=$ almostnever to $3=$ almostalways), they feel worried, bothered or nervous. ${ }^{31}$

Korean version of the ADHD Rating Scale (K-ARS)

The K-ARS was used to assess ADHD which was originally developed by DuPaul. ${ }^{32}$ The K-ARS is a scale for ADHD symptom severity composed of 18 items (9 items for 
Table 1 Measurement tools in each wave

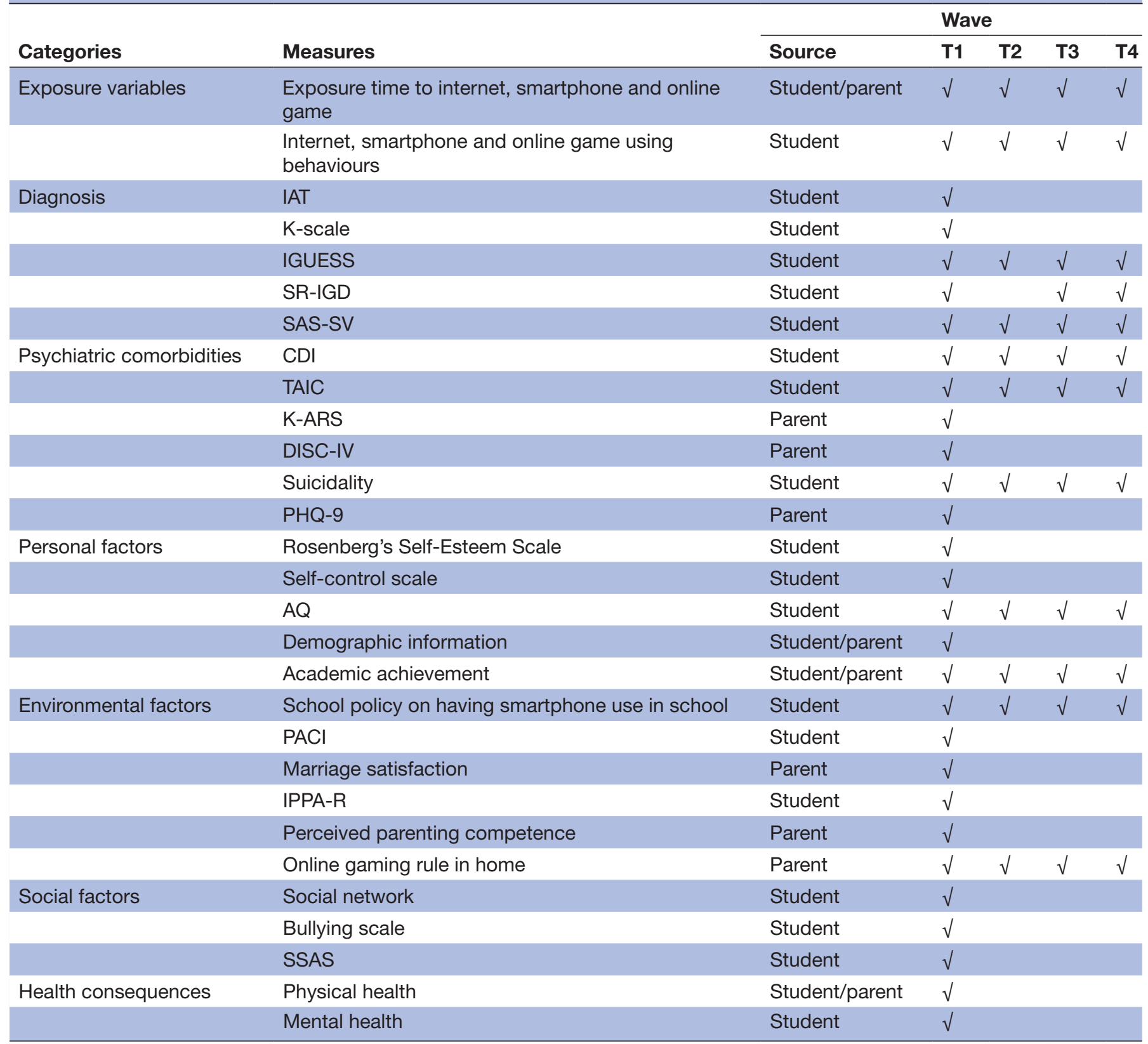

AQ, Aggression Questionnaire; CDI, Children's Depression Inventory; DISC-IV, Diagnostic Interview Schedule for Children, Version IV; IAT, Internet Addiction Test; IGUESS, Internet Game Use-Elicited Symptom Screen; IPPA-R, Revised Inventory of Parent and Peer Attachment; K-ARS, Korean version of the ADHD Rating Scale; K-scale, Korean Scale for Internet Addiction; PHQ-9, Patient Health Questionnaire; PACI, Parent-Adolescent Communication Inventory; SAS-SV, Short version of the Smartphone Addiction Scale; SR-IGD, Self-reported version of structured clinical interview for internet gaming disorder; SSAS, Social Support Appraisals Scale: Child's Subjective Appraisal of Family, Peer and Teacher Support; TAIC, Trait Anxiety Inventory for Children.

inattention and 9 items for hyperactivity) scored from as 0 (never or rarely) to 3 (very often). ${ }^{33}$

\section{Diagnostic Interview Schedule for Children, Version IV}

In the current study, the DISC-IV was administered by a trained interviewer. The DISC-IV comprised six domains of possible impairment present during a 'time in the last year when symptoms caused the most problems.' These domains are (1) getting along with parent caretakers, (2) participating in family activities, (3) participating in peer activities, (4) academic/occupational functioning, (5) relationships with teachers and (6) distress attributable to symptoms. Each set of questions has a two-part structure, the first determining whether impairment is present, and the second measuring severity or frequency. We selected an introductory module that includes demographic information (age, grade, names and ages of siblings and identification of caretakers and attachment figures). 
The remainder of the interview is organised into three modules, each containing related diagnoses (anxiety, mood and disruptive disorders). ${ }^{34}$

\section{Presence of suicidal ideation, suicide plans and suicide attempts}

The presence of suicidal ideation, suicide plans and suicide attempts is determined using the following direct questions derived from the Structured Clinical Interview for DSM-IV Axis I Disorders. Participants are asked whether they had seriously considered committing suicide, made a plan to commit suicide or attempted suicide during the past year. The presence or absence of suicidal ideation, plans and attempts is based on the subject response (yes or no). ${ }^{35}$

\section{Patient Health Questionnaire (PHQ-9)}

The PHQ-9 is a multipurpose instrument that assists in screening, diagnosing and monitoring depression severity. It incorporates the DSM-IV depression diagnostic criteria with other leading major depressive symptoms into a brief nine-item, self-report tool. Respondents rate the frequency of the symptoms over the last 2 weeks on a 4-point rating scale (not at all $=0$; several days $=1$; more than half of the days $=2$; nearly every day $=3$ ) ${ }^{36}$

\section{Rosenberg's Self-Esteem Scale}

Rosenberg's Self-Esteem Scale has 10 items, each rated on a 5-point Likert scale, ranging from 1 (strongly disagree) to 5 (strongly agree). The total score ranges from 10 to $50 . .^{37}$

\section{Self-control scale}

Self-control scale is defined by Gottfredson and Hirschi. ${ }^{38}$ The 20-item scale is divided into two self-control constructs: immediate and delayed satisfaction. Each item is rated on a 4-point scale: (4) strongly agree, (3) agree somewhat, (2) disagree somewhat and (1) strongly disagree. Higher scores indicate lower self-control.

\section{Aggression Questionnaire (AQ)}

The Buss-Perry Aggression Questionnaire is a 29-item scale that measures four aspects of human aggression and hostile personality. Participants rate each item using a 5-point Likert scale from 1 (uncharacteristic of me) to 5 (very characteristic of me) ${ }^{39}$

Social Support Appraisals Scale: Child's Subjective Appraisal of Family, Peer and Teacher Support (SSAS)

We use the SSAS to address the adolescent participants' perceived support. It is a 41-item self-report instrument developed by Dubow and Tisak. ${ }^{40}$ Items are rated on a 5-point Likert scale, from 1 (never) to 5 (always).

\section{Parent-Adolescent Communication Inventory (PACl)}

The PACI is a 40-item inventory used to assess communication in the parent-adolescent relationship. There are two forms of the inventory, one for parents and the other for adolescents. Scores range from 0 to 120, with higher scores reflecting a greater degree of parent-adolescent communication. ${ }^{41}$

The Children's Perception of Interparental Conflict Scale (CPIC)

The CPIC uses a multiple-choice format with three possible responses: true, sort of true and false. Items are scored from 1 to 3 , with 3 reflecting more negative forms of conflict and their appraisal. ${ }^{42}$

\section{Revised Inventory of Parent and Peer Attachment (IPPA-R)}

The original IPPA was developed by Armsden and Greenberg $^{43}$ to assess adolescents' perceptions of the positive and negative affective and cognitive dimensions of relationships with their parents and close friends. In particular, the inventory examines how well these figures serve as sources of psychological security. Three broad dimensions are assessed: degree of mutual trust, quality of communication and alienation. Participants are asked to rate each of the items on a 5-point Likert scale from 1 (never true) to 5 (always true). ${ }^{43}$

\section{Physical and mental health status}

We assess physical pain experiences, such as in the hand, wrist, shoulder or neck; dry eyes; drinking and smoking habits during the past year; histories of physical illness, including rheumatoid arthritis, lupus, diabetes mellitus and psychiatric illness, including depression, anxiety disorders and ADHD. We also ask about sleep hours during the week and during weekends.

\section{Sample size estimation}

We calculated the study sample size required to investigate whether overuse of internet gaming is a risk factor of IGD presence at the 2-year follow-up. For a $15 \%$ prevalence of exposure to 3 or more hours per day of internet gaming, a $5 \%$ incidence of IGD at the 2-year follow-up period, a relative risk of this risk factor for IGD of $2(R R=2.0)$, and setting $\alpha$ at 0.05 and $\beta$ at 0.20 (power of 0.80 ), a sample size of 2559 is required. Considering a dropout rate of $14 \%$, the required sample size is estimated to be almost 3000 . We expect there will be 150 adolescents at high risk for IGD (meeting three or more of the nine DSM-5 IGD criteria) among the 3000 participants at baseline, and we estimate that 75 new IGD cases will be found at the 2-year follow-up. ${ }^{44}$

\section{Strategies to minimise error and bias \\ Online assessment system}

The participants $\log$ onto the study's website with unique authentication codes provided, where they complete the online questionnaires. We use a branched algorithmic structure to enhance the confidentiality and accuracy of responses and to reduce the risk of exposing sensitive information.

\section{Training of the interviewers}

To administer the parent-guardian surveys, we recruited psychologists and nurses with Bachelor's or Master's degrees, and the research team provided them with 
training that included information on internet gaming addiction and communication skills, through a 2-day (16hours) training workshop. In addition to administering the surveys, the interviewers reported on the status of the data collection and any problems and issues arising during the interviews, at mandatory weekly meetings.

Prior to the diagnostic interviews and two times each year, all psychiatrists and clinical psychologists attend a seminar about IGD diagnosis to increase inter-rater reliability and diagnostic agreement. In the seminar, we review recent papers on IGD diagnosis and provide mock cases in a video to enhance diagnostic concordance. Also, at the end of each day of diagnostic interviews, all psychiatrists and clinical psychologists who participate in the clinical diagnosis attend a case conference to discuss any ambiguous diagnoses and make final determinations.

\section{Statistical analyses plan}

We will calculate the 2-year incidence, remission and recurrence rates of IGD, with 95\% CIs. We will investigate the cross-sectional and longitudinal associations between exposures and outcomes using a logistic or linear regression model or a generalised linear model, as appropriate. Models will include exposures to games, such as time spent playing games, types of internet games and gaming-related activities, with IGD as the outcome variable and covariates including sociodemographic, personal, social and environmental factors. Moderation tests will examine for differences in associations and mediation tests will assess indirect effects based on sociodemographic, personal, social and environmental factors.

\section{ETHICS AND DISSEMINATION}

All procedures performed in studies involving human participants were in accordance with the ethical standards of the Institutional Review Board of the Catholic University of Korea (approval number: MC140NM10085) and with the 1964 Declaration of Helsinki and its later amendments or comparable ethical standards. Written consent was received from all participants and from one of the participants, parents or caregivers following an explanation of the study, including confidentiality and freedom of choice to participate. All results will be published in relevant peer-reviewed international scientific journals and presented at conferences, nationally and internationally.

\section{DISCUSSION}

A major strength of this study is that it is conducted in a county with a particularly high burden of IGD in adolescents. Korea has a high likelihood of exposure to games because Korea plays a leading role in the development of the game industry and is one of the most highly digitalised societies in the world. It provides a high-access environment for internet gaming, including PC rooms, which have broadband internet networks and are equipped with the best performance computers in Korea. This makes Korea an optimal place to perform a cohort study to characterise IGD.

We are screening third-grade, fourth-grade and seventhgrade students for IGD using both polychotomous and dichotomous tools based on the nine DSM-5 IGD criteria. In addition, we are conducting structured interviews using DISC-IV of the parents or guardians to assess participant psychiatric comorbidities. Because the participants are early adolescents, we obtained their mental health status through their parents or guardians. Self-reports can include false or insincere reports, making evaluation of the IGD risk factors less accurate. To reduce this effect, we have added a reverse scale to each self-report questionnaire.

Another strength of this study is that the clinical diagnosis of IGD based on the DSM- 5 is performed by qualified mental health specialists. Most prior studies have not been able to also get a true clinical diagnosis along with the screening tool scores, so this study will provide needed validity data. Participants included those considered positive for IGD, defined as scores of 6 or higher on IGUESS, as well as a reference group of a random selection of $10 \%$ of the participants. We have constructed two subcohorts from those who have participated in the clinical diagnosis: a representative and a high-risk cohort. The representative subcohort consists of $10 \%$ randomly selected adolescents from the full cohort. They participate in the clinical diagnostic interview every year in order to estimate the false negative rate and to evaluate the clinical course of IGD, observed via the diagnostic interviews. The high-risk subcohort comprised participants meeting three or more of the nine IGD criteria, based on the clinical diagnostic interviews at baseline and each follow-up wave. The high-risk subcohort is similar to a dynamic cohort. The suggested diagnostic criteria of IGD in DSM-5 is to meet five or more of the nine IGD criteria; however, we plan to follow those adolescent participants meeting three or more clinical diagnostic interview in any year in order to investigate the course of IGD at syndromal and subsyndromal levels.

This study aims to clarify the natural and clinical courses of IGD based on the DSM-5 suggested diagnostic criteria in order to empirical validate the criteria and to evaluate the risk and predictive factors in adolescents based on the IGD diagnostic criteria. This study will contribute to establishing standardised IGD diagnostic criteria and to providing scientific evidence regarding the proposed DSM-5 IGD diagnostic criteria.

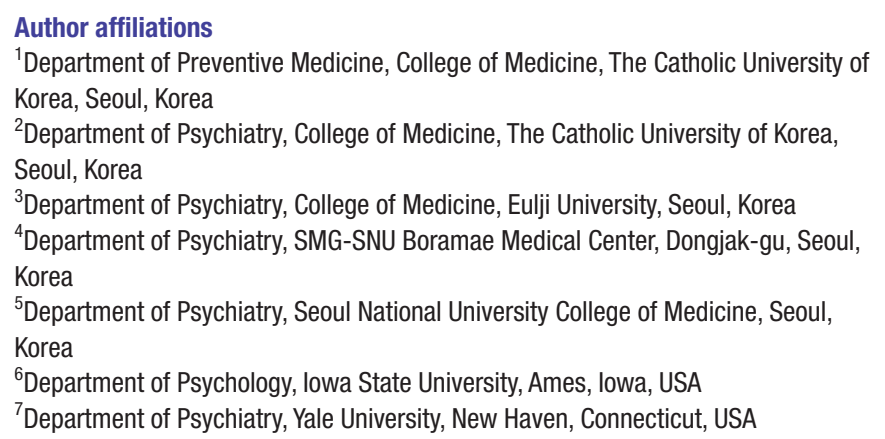

${ }^{1}$ Department of Preventive Medicine, College of Medicine, The Catholic University of Korea, Seoul, Korea

${ }^{2}$ Department of Psychiatry, College of Medicine, The Catholic University of Korea, Seoul, Korea

${ }^{3}$ Department of Psychiatry, College of Medicine, Eulji University, Seoul, Korea ${ }^{4}$ Department of Psychiatry, SMG-SNU Boramae Medical Center, Dongjak-gu, Seoul, Korea

${ }^{5}$ Department of Psychiatry, Seoul National University College of Medicine, Seoul, Korea

${ }^{6}$ Department of Psychology, lowa State University, Ames, lowa, USA

${ }^{7}$ Department of Psychiatry, Yale University, New Haven, Connecticut, USA 
Contributors $\mathrm{HJ}$ conducted the analyses and led the writing of the manuscript. HWY guided and supervised the writing of the manuscript. HWY, HJ and S-JJ developed and proposed the basic idea of the study. HWY, HJ, S-JJ, EK, HJS and $\mathrm{H}$-hH contributed to conducting the study. S-YL, HKL, Y-SK, S-yB and J-SC conducted diagnostic interviews. B-NK guided to performance DISC assessment. DAG advised to construct study design. DAG and MNP reviewed scientific and proof-reading of the manuscript. All authors contributed editorial comments on the manuscript.

Funding This study was supported by a grant from the Korean Mental HealthTechnology R\&D Project, Ministry of Health and Welfare, Republic of Korea (HM14C2603).

\section{Competing interests None declared.}

Patient consent All procedures performedin studies involving human participants were in accordance with the ethicalstandards of the Institutional Review Board of The Catholic University of Korea(approval number: MC140NM10085) and withthe 1964 Helsinki declaration and its later amendments or comparable ethicalstandards. Written consent was received from all participants and from one ofthe participants' parents or caregivers following an explanation of the study,including confidentiality and freedom of choice to participate.

Ethics approval The Institutional Review Board of the Catholic University of Korea (approval no: MC140NM10085).

Provenance and peer review Not commissioned; externally peer reviewed.

Open Access This is an Open Access article distributed in accordance with the Creative Commons Attribution Non Commercial (CC BY-NC 4.0) license, which permits others to distribute, remix, adapt, build upon this work non-commercially, and license their derivative works on different terms, provided the original work is properly cited and the use is non-commercial. See: http://creativecommons.org/ licenses/by-nc/4.0/

(C) Article author(s) (or their employer(s) unless otherwise stated in the text of the article) 2017. All rights reserved. No commercial use is permitted unless otherwise expressly granted.

\section{REFERENCES}

1. Entertainment_software_association. 2012 Essential facts about the computer and video game industry. 2012 http://wwwtheesacom/ facts/pdfs/ESA_EF_2012pdf

2. Wan CS, Chiou WB. Why are adolestants addicted to online gaming? An interview study in Taiwan. The impact of the Internet, multimedia and virtual reality on behavior and society 2006;9:762-6.

3. Wood RT, Griffiths MD. A qualitative investigation of problem gambling as an escape-based coping strategy. Psychol and Psychother 2007;80:107-25.

4. Kuss DJ, Griffiths MD, Karila L, et al. Internet addiction: a systematic review of epidemiological research for the last decade. Curr Pharm Des 2014;20:4026-52.

5. Boot WR, Kramer AF, Simons DJ, et al. The effects of video game playing on attention, memory, and executive control. Acta Psychol 2008;129:387-98.

6. Witt EA, Massman AJ, Jackson LA. Trends in youth's videogame playing, overall computer use, and communication technology use: the impact of self-esteem and the big five personality factors. Comput Human Behav 2011;27:763-9.

7. Chan PA, Rabinowitz T. A cross-sectional analysis of video games and attention deficit hyperactivity disorder symptoms in adolescents. Ann Gen Psychiatry 2006;5:16.

8. Desai RA, Krishnan-Sarin S, Cavallo D, et al. Video-gaming among high school students: health correlates, gender differences, and problematic gaming. Pediatrics 2010;126:e1414-1424.

9. Van Rooij AJ, Schoenmakers TM, Vermulst AA, et al. Online video game addiction: identification of addicted adolescent gamers. Addiction 2011;106:205-12.

10. BarnettJaC .Mark virtually real: a psychological perspective on massively multiplayer online games.Review of General Psychology2010;14:167-79.

11. Union IT. World Telecommunication/ ICT Indicators Database. 17th edition, 2013.

12. Service KSI. Statistics on internet use.National Information Society Agency (2011) internet addiction survey. Seoul, South Korea: Agency NIS Agency NIS.

13. Ministry of Culture $S$, and Tourism. Introduction of regulations of internet game in Korea, 2014.
14. Choo $\mathrm{H}$, Gentile DA, Sim $\mathrm{T}$, et al. Pathological video-gaming among singaporean youth. Ann Acad Med Singapore 2010;39:822-9.

15. Lemmens JS, Valkenburg PM, Gentile DA. The Internet Gaming Disorder Scale. Psychol Assess 2015;27:567-82.

16. Gentile D. Pathological video-game use among youth ages 8 to 18 : a national study. Psychol Sci 2009;20:594-602.

17. Lam LT, Peng ZW, Mai JC, et al. Factors associated with Internet addiction among adolescents. Cyberpsychol Behav 2009;12:551-5.

18. American Psychiatric Association. Diagnostic and Statistical Manual of Mental Disorders (DSM-5), 2013.

19. Petry NM, Rehbein F, Gentile DA, Lemmens JS, et al. An international consensus for assessing internet gaming disorder using the new DSM-5 approach. Addiction 2014;109:1399-406.

20. Griffiths MD, van Rooij AJ, Kardefelt-Winther D, et al. Working towards an international consensus on criteria for assessing internet gaming disorder: a critical commentary on Petry et al. (2014). Addiction 2016;111:167-75.

21. Kardefelt-Winther D, Heeren A, Schimmenti A, et al. How can we conceptualize behavioural addiction without pathologizing common behaviours? Addiction 2017 (Epub ahead of print 16 Feb 2017)

22. Starcevic V. Tolerance and withdrawal symptoms may not be helpful to enhance understanding of behavioural addictions. Addiction 2016;111:1307-8.

23. Ko $\mathrm{CH}$, Yen JY, Chen $\mathrm{SH}$, et al. Evaluation of the diagnostic criteria of Internet gaming disorder in the DSM-5 among young adults in Taiwan. J Psychiatr Res 2014;53:103-10.

24. Kim YS, Cheon KA, Kim BN, et al. The reliability and validity of Kiddie-Schedule for Affective Disorders and Schizophrenia-Present and Lifetime Version- Korean version (K-SADS-PL-K). Yonsei Med J 2004:45:81-9.

25. Young KS. The research and controversy surrounding internet addiction. Cyberpsychol Behav 1999;2:381-3.

26. Kim DI JY, Lee EA, Kim DM, et al. Development of internet addiction proneness scale - short form (KS-scale). Korean J Counseling 2008;9:1703-22

27. Koo HJ, Han DH, Park SY, et al. The Structured Clinical Interview for DSM-5 Internet Gaming Disorder: Development and Validation for Diagnosing IGD in Adolescents. Psychiatry Investig 2017;14:21-9.

28. Kwon M, Kim DJ, Cho H, et al. The smartphone addiction scale: development and validation of a short version for adolescents. PLoS One 2013;8:e83558.

29. Kovacs M, Feinberg TL, Crouse-Novak MA, et al. Depressive disorders in childhood. I. A longitudinal prospective study of characteristics and recovery. Arch Gen Psychiatry 1984;41:229-37.

30. Cho S. CJ Development of state - Trait Anxiety Scale for Korean Children. J Med Seoul Natinal University 1989;14:150-7.

31. Spielberger CD. Manual for the state: Trait Anxiety Inventory for Children. Palo Alto: Consulting Psychologist Press, 1972.

32. Gj. D. Parent and teacher ratings of ADHD symptoms: psychometric properties in a community-based sample. J Clin Child Psychol 1990;20:245-53.

33. Kim JW, Park KH, Cheon KA, et al. The child behavior checklist together with the ADHD rating scale can diagnose ADHD in Korean community-based samples. Can J Psychiatry 2005;50:802-5.

34. Shaffer D, Fisher P, Lucas CP, et al. NIMH Diagnostic Interview Schedule for Children Version IV (NIMH DISC-IV): description, differences from previous versions, and reliability of some common diagnoses. J Am Acad Child Adolesc Psychiatry 2000;39:28-38.

35. First MB, SpitzerRL, Gibbon M, et al. Structured Clinical Interview for DSM-IV-TR Axis I Disorders, Research Version, Research Edition. New York: Biometrics Research, New York State Psychiatric Institute, 2002.

36. Kroenke K, Spitzer RL, Williams JB. The PHQ-9: validity of a brief depression severity measure. J Gen Intern Med 2001;16:606-13.

37. Rosenberg M. Society and the adolescent self-image. Princeton University Press: Princeton, NJ, 1965

38. Gottfredson MR, Hirschi T. A generaltheory of crime. Stanford University Press: Stanford, CA, 1990.

39. Buss $A H$, Perry M. The aggression questionnaire. J Pers Soc Psychol 1992;63:452-9.

40. Dubow EF, Tisak J. The relation between stressful life events and adjustment in elementary school children: the role of social support and social problem-solving skills. Child Dev 1989;60:1412-23.

41. Bienvenu MJ. A parent-adolescent communicaiton inventory. Saluda, NC: Family Life publictions, 1969.

42. Grych JH, Seid M, Fincham FD. Assessing marital conflict from the child's perspective: the children's perception of interparental conflict scale. Child Dev 1992;63:558-72.

43. Armsden GC, Greenberg MT. The inventory of parent and peer attachment: Individual differences and their relationship 
to psychological well-being in adolescence. $J$ Youth Adolesc 1987;16:427-54.
44. Gentile DA, Choo H, Liau A, et al. Pathological video game use among youths: a two-year longitudinal study. Pediatrics 2011;127:e319-29. 